\title{
Generating All Simple Convexly-Drawable Polar Symmetric 6-Venn Diagrams
}

\author{
Khalegh Mamakani and Frank Ruskey \\ Dept. of Computer Science, University of Victoria, Canada.
}

\begin{abstract}
An $n$-Venn diagram consists of $n$ curves drawn in the plane in such a way that each of the $2^{n}$ possible intersections of the interiors and exteriors of the curves forms a connected non-empty region. A Venn diagram is convexly-drawable if it can be drawn with all curves convex and it is simple if at most two curves intersect at any point. A Venn diagram is called polar symmetric if its stereographic projection about the infinite outer face is isomorphic to the projection about the innermost face. We outline an algorithm that shows there are exactly 406 simple convexly drawable polar-symmetric 6 -Venn diagrams.
\end{abstract}

Key words: Venn diagram, polar-symmetry

\section{Introduction}

Named after John Venn(1834 - 1923), who used diagrams of overlapping circles to represent propositions [10], Venn diagrams are commonly used in set theory to visualize the relationship between different sets. When talking about Venn diagrams, the traditional three circles diagram with 3 -fold symmetry often comes to mind (Figure 1(a)). Although it is not possible to use circles to draw Venn diagrams of more than 3 sets, more than 3 sets can be represented if the curves of the Venn diagram are other simple closed curves. Figure 1(b) shows a 5set Venn diagram composed of 5 congruent ellipses which was discovered by Grünbaum[5], and Figure 1(c) shows a 7-set Venn diagram with 7-fold rotational symmetry called Adelaide which was discovered independently by Grünbaum [7] and Edwards [3].

Intensive research has been done recently on generating and drawing Venn diagrams of more than three sets, particularly in regard to symmetric Venn diagrams, which are those where rotating the diagram by $360 / n$ degrees results in the same diagram. Henderson considered rotationally symmetric Venn diagrams and he showed that they could exist only prime number of curves [9]. Griggs, Killian, and Savage published a constructive method for producing symmetric Venn diagrams with a prime number of curves [8]. Venn diagrams exist for any number of curves and several constructions of them are known [12].

Another type of symmetry, introduced by Grübaum [6], is called polarsymmetry which can be imagined by first projecting the diagram onto the surface of a sphere with the regions corresponding to the full and empty sets at the 
north and south poles, respectively. The Venn diagram is polar-symmetric if it is invariant under polar flips, meaning that the north and south hemispheres are congruent. In other words, for a polar-symmetric Venn diagram on the plane turning the diagram inside-out (the innermost face becomes the outermost face) gives the same Venn diagram. Note that all three Venn diagrams of Figure 1 are polar-symmetric, as well as being rotationally symmetric.

The only other attempt that we know of to exhaustively list some interesting class of 6-Venn diagrams occurs in the the work of Jeremy Carroll, who discovered that there are precisedly 126 such Venn diagrams where all curves can be drawn as triangles [2]. He used a brute force search algorithm for all possible face sizes of a Venn diagram. However, the problem of generating polar-symmetric six-set Venn diagrams has not been studied before. In this paper we are restricting our attention to the special (and most studied) class of Venn diagrams that are both simple and monotone (drawable with convex curves). We introduce two representations of these diagrams. Inspired by Carroll's work, an algorithm for generating all possible simple monotone polar-symmetric six-set Venn diagrams is developed - an algorithm which determines that there are exactly 406 simple monotone polar-symmetric 6 -Venn diagrams.

Although our results are oriented towards 6-Venn diagrams, they could in principle be applied to general $n$-Venn diagrams, but the computations will quickly become prohibitive. Nevertheless, we believe that the data structures and ideas introduced here (i.e., the representations) will be useful in further investigations, and in particular to resolving one of the main outstanding open problems in the area of Venn diagrams: is there a simple 11-Venn diagram? We intend to use the data structures proven useful here to attack a restricted, but natural, version of that problem: is there a simple monotone polar-symmetric 11-Venn diagram?

The study of symmetric Venn diagrams in interesting not only because symmetry is core aspect of mathematical enquiry, but also because we are often led to diagrams of great inherent beauty. Furthermore, the geometric dual of a simple Venn diagram is a maximal planar spanning subgraph of the the hypercube, and so results about Venn diagrams often have equivalent statements as results about the hypercube.

The remainder of paper is organized as follows. In the Section 2 we introduce basic definitions. Representations of simple monotone Venn diagrams are explained in Section 3. The generating algorithm and results are explained in the last two sections.

\section{Basic Definitions}

A closed curve in the plane is simple if it doesn't intersect itself. Each simple closed curve decomposes the plane into two connected regions, the interior and the exterior. An $n$-Venn diagram is a collection of $n$ finitely intersecting simple closed curves $\mathcal{C}=\left\{C_{0}, C_{1}, \ldots, C_{n-1}\right\}$ in the plane, such that there are exactly $2^{n}$ nonempty and connected regions of the form $X_{0} \cap X_{1} \cap \cdots \cap X_{n-1}$, where 


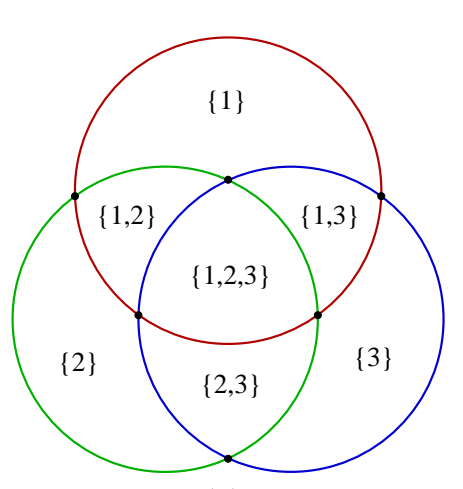

(a)

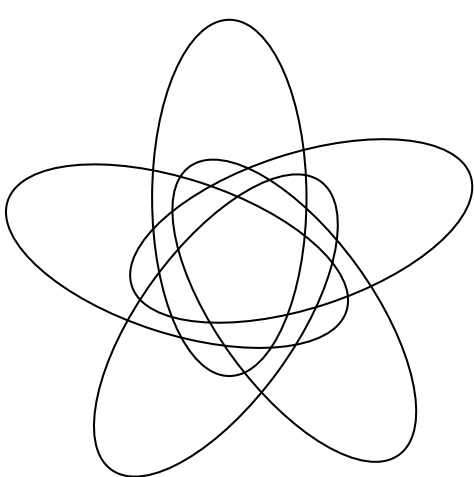

(b)

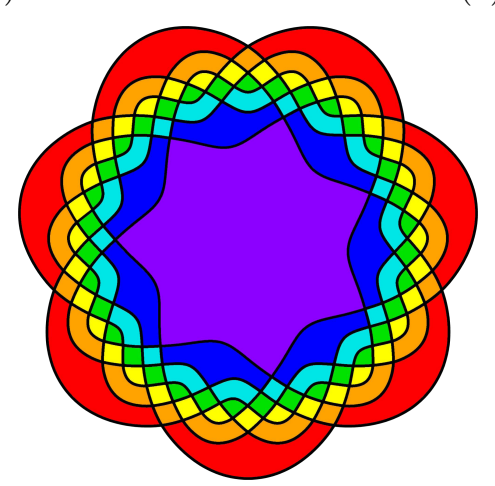

(c)

Fig. 1. (a) A 3-Venn diagram whose curves are circles. (b) A 5-Venn diagram whose curves are ellipses. (c) A symmetric 7-Venn known as "Adelaide."

$X_{i}$ is either the unbounded open exterior or open bounded interior of curve $C_{i}$. Each connected region corresponds to a subset of the set $\{0,1, \ldots, n-1\}$. Two Venn diagrams are isomorphic if one of them can be changed into the other or its mirror image by a continuous transformation of the plane.

A $k$-region in a diagram is a region that is in the interior of precisely $k$ curves. In an $n$-Venn diagram, each $k$-region corresponds to a $k$-element subset of a set with $n$ elements. So, there are $\left(\begin{array}{l}n \\ k\end{array}\right) k$-regions. A Venn diagram is monotone if every $k$-region is adjacent to both some $(k-1)$-region (if $k>0$ ) and also to some $(k+1)$-region (if $k<n)$. A diagram is monotone if and only if it is drawable with each curve convex [1]. A simple Venn diagram is one in which exactly two curves cross each other at each intersection point. Figure 2 shows a simple monotone 6-Venn diagram.

Consider a Venn diagram as being projected onto the surface of a unit sphere where the empty region of the diagram encloses the north pole of the sphere and the innermost region contains the south pole. A cylindrical projection of the 


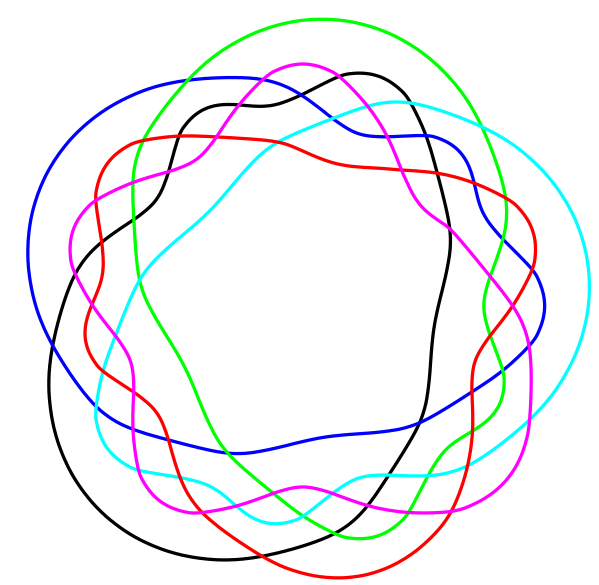

Fig. 2. A simple monotone 6-Venn diagram.

Venn diagram can be obtained by mapping the surface of sphere to a $2 \pi$ by 2 rectangle on the plane, where the equator of the sphere maps to a horizontal line of length $2 \pi$ and the north and south pole of the sphere are mapped to the top and bottom sides of the rectangle respectively. In this representation, the top of cylinder represents the empty region and bottom of cylinder represents the innermost region. A Venn diagram is said to be polar symmetric if it is invariant under polar flips. In the cylindrical representation the polar flip is equivalent to turning the cylinder upside-down. For a Venn diagram on the plane, a polar flip is equivalent to turning the diagram inside-out, with the innermost face becoming the outermost. Figure 3 shows the cylindrical representation of the polar symmetric 6-Venn diagram shown in Figure 2. It is known that there are exactly 6 simple monotone 7 -Venn diagrams with rotational and polar symmetry $[4],[3]$.

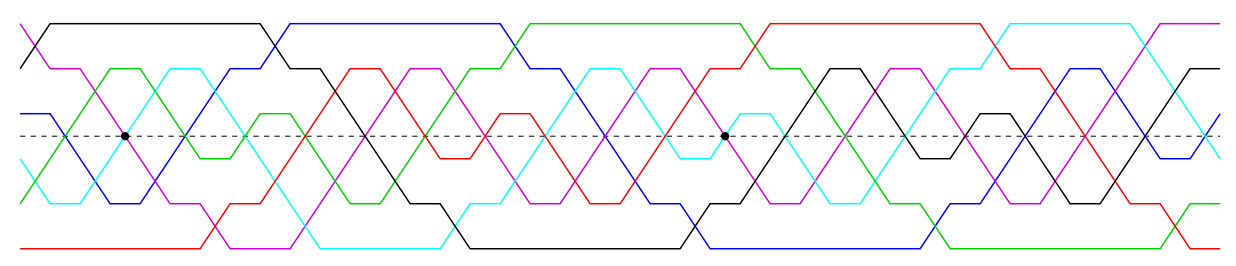

Fig. 3. Cylindrical representation of the 6-Venn diagram of Figure 2.

A simple Venn diagram can be viewed as a planar graph where the intersection points of the Venn diagram are the vertices of the graph and the sections of 
the curves that connect the intersection points are the edges of the graph. For a planar graph with $f$ faces, $v$ vertices and $e$ edges, Euler's formula states that $f+v=e+2$. A graph of an $n$-Venn diagram has $2^{n}$ faces. In a simple Venn diagram each vertex of this graph has degree 4 ; i.e. $e=2 v$, so a simple $n$-Venn diagram has $2^{n}-2$ vertices.

\section{Representing Venn Diagrams}

In this section we introduce two representations for simple monotone Venn diagrams. First we discuss the binary matrix representation where each 1 in the matrix represents an intersection point of the corresponding Venn diagram. Having the matrix representation of a diagram, it is easy to check if it is a Venn diagram or not. In the second part we show how to represent simple monotone Venn diagrams using compositions. We use this representation to find all candidate diagrams. Then we filter non-Venn diagrams using the matrix representation.

\subsection{Matrix representation}

For a simple monotone $n$-Venn, every 1-region is adjacent to the empty region. So the empty region surrounds a "ring" of $\left(\begin{array}{l}n \\ 1\end{array}\right)$ 1-regions. Since each region is started by one intersection point and ended by another one, there are $\left(\begin{array}{l}n \\ 1\end{array}\right)$ intersection points in the first ring. Similarly, every 2 -region is adjacent to at least one 1-region. So, there are $\left(\begin{array}{l}n \\ 2\end{array}\right)$ 2-regions that form a second ring surrounded by the first ring and which contains $\left(\begin{array}{l}n \\ 2\end{array}\right)$ intersection points. In general, in a simple monotone $n$-Venn diagram, there are $n-1$ rings of regions, where all regions in a ring are enclosed by the same number of curves and every region in ring $i$, $1 \leq i \leq n-1$, is adjacent to at least one region in ring $i-1$ and also to at least one region in ring $i+1$. The number of intersection points in the $i^{\text {th }}$ ring is the same as the number of regions, which is $\left(\begin{array}{l}n \\ i\end{array}\right)$. The rings have different colors in Figure 1(c).

Thus a simple monotone $n$-Venn diagram can be represented by a $n$ by $m$ binary matrix, $m \leq 2^{n}-2$, where each 1 in the matrix represents an intersection point in the Venn diagram. A Venn matrix has the following properties :

- There are $\left(\begin{array}{c}n \\ i\end{array}\right) 1$ 's in the $i^{\text {th }}$ row of the matrix, $1 \leq i \leq n-1$.

- There are no two adjacent 1's in any row or column of the matrix.

- A valid matrix must represent exactly $2^{n}-2$ distinct regions of the corresponding Venn diagram. The two other regions are the outermost and the innermost regions. Figure 4 shows the matrix representation of the Venn diagram of Figures 2 and 3.

The rank of a region of a Venn diagram is defined by $\sum_{i=0}^{n-1} 2^{i} X_{i}$ where $x_{i}=1$ if curve $i$ encloses the region and $x_{i}=0$ otherwise. Given a matrix representation $P$, we need to check that no two ranks are the same to check if it represents a valid Venn diagram. 


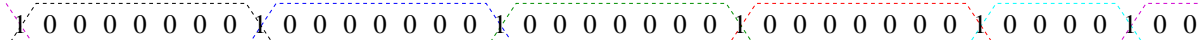
00101010000101010001010100010101000101010 $010101010101010101010101010101010 \times 010101$ 1010100010101000101010001010100010101000 $\begin{array}{lllllllllllllllllllllllllllllllllllllll}0 & 0 & 0 & 0 & 0 & 0 & 1 & 0 & 0 & 1 & 0 & 0 & 0 & 0 & 1 & 0 & 0 & 0 & 0 & 0 & 0 & 0 & 1 & 0 & 0 & 0 & 0 & 0 & 0 & 0 & 1 & 0 & 0 & 0 & 0 & 0 & 0 & 0 & 1\end{array}$

Fig. 4. Matrix representation of the 6-Venn diagram of Figures 2 and 3.

For a given matrix $P$, suppose vector $C=\left[c_{0}, c_{1}, \cdots, c_{n-1}\right]$ represents the curve labels of the corresponding diagram in cylindrical representation where $c_{0}$ is the label of the outmost curve and $c_{n-1}$ is the label of the innermost curve. Then a region at ring $i, 1 \leq i \leq n-1$, is enclosed by curves $c_{0}, \cdots, c_{i-1}$ and the rank of the region is $\sum_{k=0}^{i-1} 2^{k} c_{k}$. To get the curve labels for each region we need to update $C$ based on the entries of matrix $P$ at each column. For a given column $j$ of the matrix, each entry of 1 represents an intersection point. So for each row $i$, if $p_{i j}=1$ then we need to exchange $c_{i}$ and $c_{i+1}$ to get the next curve labels. Starting from left to right with $C=[0,1, \cdots, n-1]$ as the initial curve labels, then we can compute the rank of each region. Matrix $P$ represents a valid Venn diagram if we get exactly $2^{n}-2$ regions with distinct ranks and $C=[0,1, \cdots, n-1]$ at the end.

\subsection{Compositions}

In this part we introduce the other representation we use to generate Venn diagrams. In this representation, we use a sequence of non-negative integers to show the size of faces in each ring and and also to specify the position of intersection points of the next ring.

Definition 1. Let $a_{1}, a_{2}, \cdots, a_{k}$ be non-negative integers such that :

$$
\sum_{i=1}^{k} a_{i}=n
$$

Then $\left(a_{1}, a_{2}, \cdots, a_{k}\right)$ is called a composition of $n$ into $k$ parts or a $k$-composition of $n$.

In a simple monotone $n$-Venn diagram there are $\left(\begin{array}{c}n \\ i+1\end{array}\right)$ intersection points at ring $i+1$ that are distributed among $\left(\begin{array}{l}n \\ i\end{array}\right)$ intersection points at ring $i$. So if we pick a particular point at ring $i$ as the reference point, then we can specify the exact location of points at ring $i+1$ using a composition of $\left(\begin{array}{c}n \\ i+1\end{array}\right)$ into $\left(\begin{array}{c}n \\ i\end{array}\right)$ parts.

Definition 2. Let $\mathcal{C}(n, k)$ be the set of all compositions of $n$ into $k$ parts. For a simple monotone $n$-Venn diagram $V$, starting from an arbitrary point of the first ring, we label the intersection points of $V$ from 1 to $2^{n}-2$ in clockwise 
direction. Let $\ell_{i}$ denote the label of the starting point at ring $i$. The composition representation $P$ of $V$ is a set of $n-2$ pairs of form $\left\langle\ell_{i}, c_{i}\right\rangle$, where

$$
\ell_{1}=1, \ell_{i}=\ell_{i-1}+\left(\begin{array}{c}
n \\
i-1
\end{array}\right), c_{i} \in \mathcal{C}\left(\left(\begin{array}{c}
n \\
i+1
\end{array}\right),\left(\begin{array}{c}
n \\
i
\end{array}\right)\right)
$$

Figure 5 shows the composition representation of the 6-Venn diagram of Figure 2 and 3 .

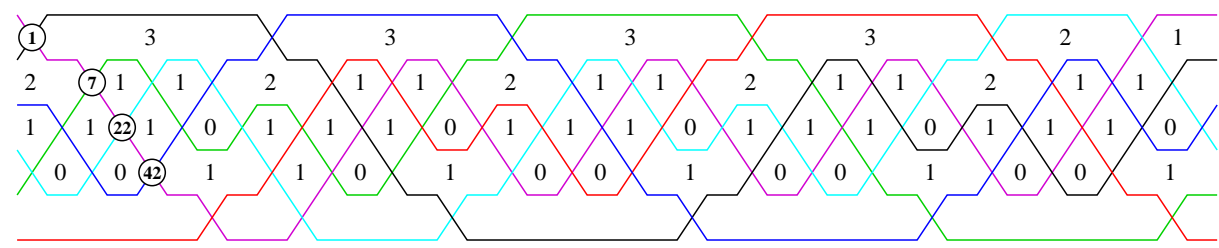

Fig. 5. Composition representation of the 6-Venn diagram of Figures 2 and 3.

We now list several observations that will help us cut down on the size of the search space.

Observation 1 For any simple monotone $n$-Venn diagram $V$, the largest part of $c_{i}$ in the composition representation is $n-i-1$.

Proof. A region at ring $i$ is enclosed by $i$ curves above its starting and ending points. Since the size of a region is at most $n$ and no two edges belong to the same curve [11], at most $n-i$ remaining curves can be used to shape the region. As shown in Figure 6, to put $p$ intersection points between the to end points of the region on the next ring, we need $p+1$ curves, $p-1$ curves for the bottom side and two curves for the left and right sides. So, $p \leq n-i-1$.

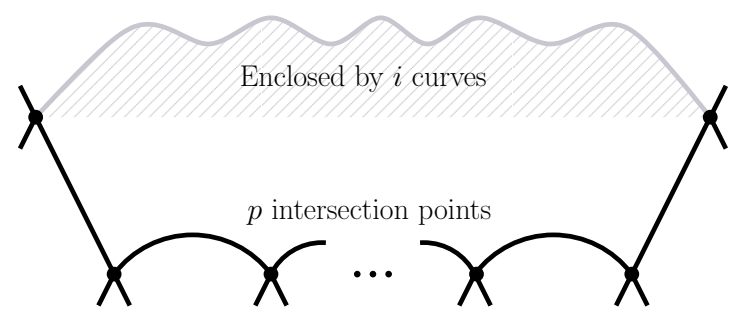

Fig. 6. Largest part of the composition at level $i$. 
Observation 2 In the composition representation of any simple monotone Venn diagram with more than 3 curves, there are no two non-adjacent 1's in $c_{1}$.

Proof. Suppose, there is a such Venn diagram $\mathcal{V}$, then the first ring of the Venn diagram will be like Figure 7 , where regions $A$ and $D$ correspond to non-adjacent 1 's in the composition and $A \neq D$. Then $A \cap D=\emptyset$ which contradicts the assumption that $\mathcal{V}$ is a Venn diagram. So in the first ring composition there are at most two 1's which must be adjacent.

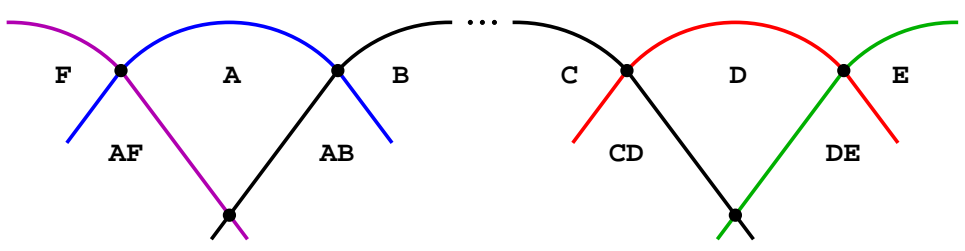

Fig. 7. Non-Adjacent 1's in the first ring composition.

Observation 3 There are no two faces of size 3 adjacent to another face of size 3 in a simple monotone $n-$ Venn diagram $\mathcal{V}$.

Proof. There are only two cases, shown in Figure 8, that two faces of size 3 could be adjacent to a single face of size 3 . However, both cases result in a two part disconnected region(the shaded region) which contradicts the fact that $\mathcal{V}$ is a Venn diagram.

Observation 4 There are no two consecutive 0's in $c_{2}$ for the composition representation of any simple monotone Venn diagram.

Proof. By observation 3

Definition 3. Let $r_{1}, r_{2} \in \mathcal{C}(n, k)$ be two composition of of $n$ into $k$ parts. $r_{1}$ and $r_{2}$ are rotationally distinct if it is not possible to get $r_{2}$ from any rotation of $r_{1}$ or its reversal.

Theorem 5. Let $\mathcal{F}_{n}$ denote the set of all rotationally distinct compositions of $\left(\begin{array}{l}n \\ 2\end{array}\right)$ into $n$ parts such that for any $r \in \mathcal{F}_{n}$ there are no two non-adjacent parts of 1 and all parts are less than or equal to $n-2$. Then all simple monotone $n$-Venn diagrams can be generated from composition representations where the first composition $c_{1} \in \mathcal{F}_{n}$.

Proof. Given a simple monotone $n$-Venn diagram, suppose we get the composition representation $P$ of $V$ by picking a particular intersection point $x$ in the first ring as the reference point. Now let $P^{\prime}$ be another representation of $V$ using 
any other intersection point different than $x$ as the reference point. It is clear that $c_{1}^{\prime}$ in $P^{\prime}$ is a rotation of $c_{1}$ in $P$. Also for any composition representation $P^{\prime \prime}$ of the mirror of $V$ the first composition $c_{1}^{\prime \prime}$ in $P^{\prime \prime}$ is a rotation of the reversal of $c_{1}$. By the observations 1 and 2 the largest part of $c_{1}$ is $n-2$ and there are no two non-adjacent 1's in $c_{1}$. Therefore, there is a composition $c \in \mathcal{F}_{n}$ which is rotationally identical to $c_{1}$.

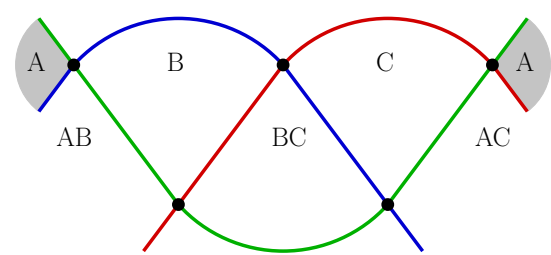

(a)

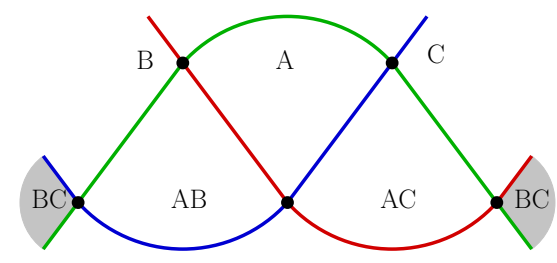

(b)

Fig. 8. Possible cases for two faces of size 3 being adjacent to a single face of size 3.

\section{Generating Algorithm}

Given the upper/lower half of the cylindrical representation of a polar symmetric Venn diagram, one can generate the whole diagram by creating a copy of the given half, turning it upside down and rotating it until the two parts match together. So, to generate a polar symmetric monotone Venn diagram, we need only to generate the first $\left\lceil\frac{n-2}{2}\right\rceil$ compositions.

Two halves of the diagram can match only if gluing them using the intersection points doesn't create any faces of size 2. Given the last composition of the upper half, for each positive part $a_{j}$ there are $a_{j}-1$ edges that bound the corresponding face from the bottom and there is a gap between two faces corresponding to two consecutive parts of the composition. So, we can map the composition to a bit-string where each 1 represents a bounding edge of a face and each 0 represents the gap between two faces. The length of bit-string is the same as the sum of all parts of the composition. In other words the composition $\left(a_{1}, a_{2}, \cdots, a_{k}\right)$ is mapped to the following bit-string.

$$
\overbrace{11 \cdots 1}^{a_{1}-1 \text { bits }} 0 \overbrace{11 \cdots 1}^{a_{2}-1 \text { bits }} 0 \cdots 0 \overbrace{11 \cdots 1}^{a_{k}-1 \text { bits }} 0
$$

We can find all matching of the two halves by computing the bitwise and of the bit-string and its reverse for all left rotations of the reverse bit-string. Any result other than 0 means that there is at least one face of size 2 in the middle. Then for each matching we compute the matrix representation of the resulting 
diagram. The matrix can be obtained by sweeping the compositions from left to right and computing the position of each intersection point. Checking each resulting matrix for all compositions gives us all possible polar symmetric Venn diagram.

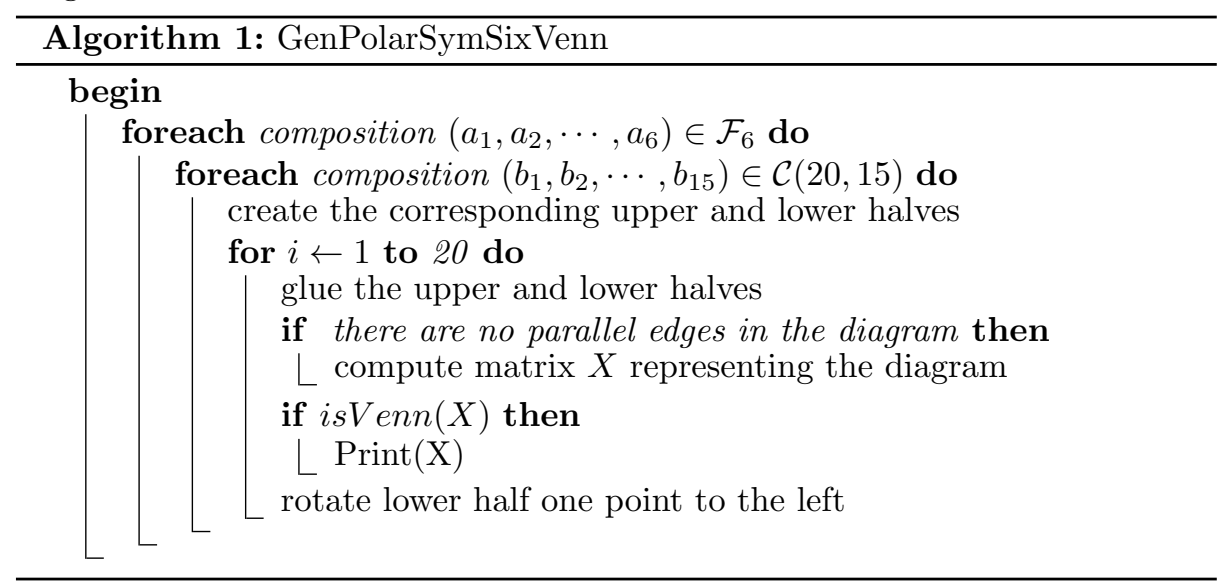

\section{5 results}

Using the exhaustive search we found 406 simple monotone polar symmetric 6 -Venn diagrams. Table 1 shows the number of Venn diagrams for each particular composition of the first level. Figure 9 shows one Venn diagram for each of those 29 compositions that have at least one Venn diagram. Except for three cases, for each pair of the first and second level compositions there is at most one Venn diagram. Each of the other three pairs give exactly two Venn diagrams. Renderings of each of the 406 diagrams may be found at the website http://webhome.cs.uvic.ca/ ${ }^{\sim}$ ruskey/Publications/SixVenn/SixVenn.html.

\section{References}

1. B. Bultena, B. Grünbaum, F. Ruskey, "Convex Drawings of Intersecting Families of Simple Closed Curves," 11th Canadian Conference on Computational Geometry, 1999, 18-21.

2. J. Carroll, "Drawing Venn triangles," Technical Report HPL-2000-73, HP Labs (2000).

3. A. W. F. Edwards, "Seven-set Venn Diagrams with Rotational and Polar Symmetry", Combinatorics, Probability, and Computing, 7 (1998) 149-152.

4. T. Cao, K. Mamakani and F. Ruskey, "Symmetric Monotone Venn Diagrams with Seven Curves," Fifth internationl conference on Fun with Algorithms, Ischia Island Italy, 2010. LNCS 6099, 331-342.

5. B. Grünbaum, "Venn Diagrams and Independent Families of Sets", Mathematics Magazine, Jan-Feb 1975, 13-23.

6. B. Grünbaum, "Venn Diagrams I", Geombinatorics, Volume I, Issue 4, (1992) 5-12. 


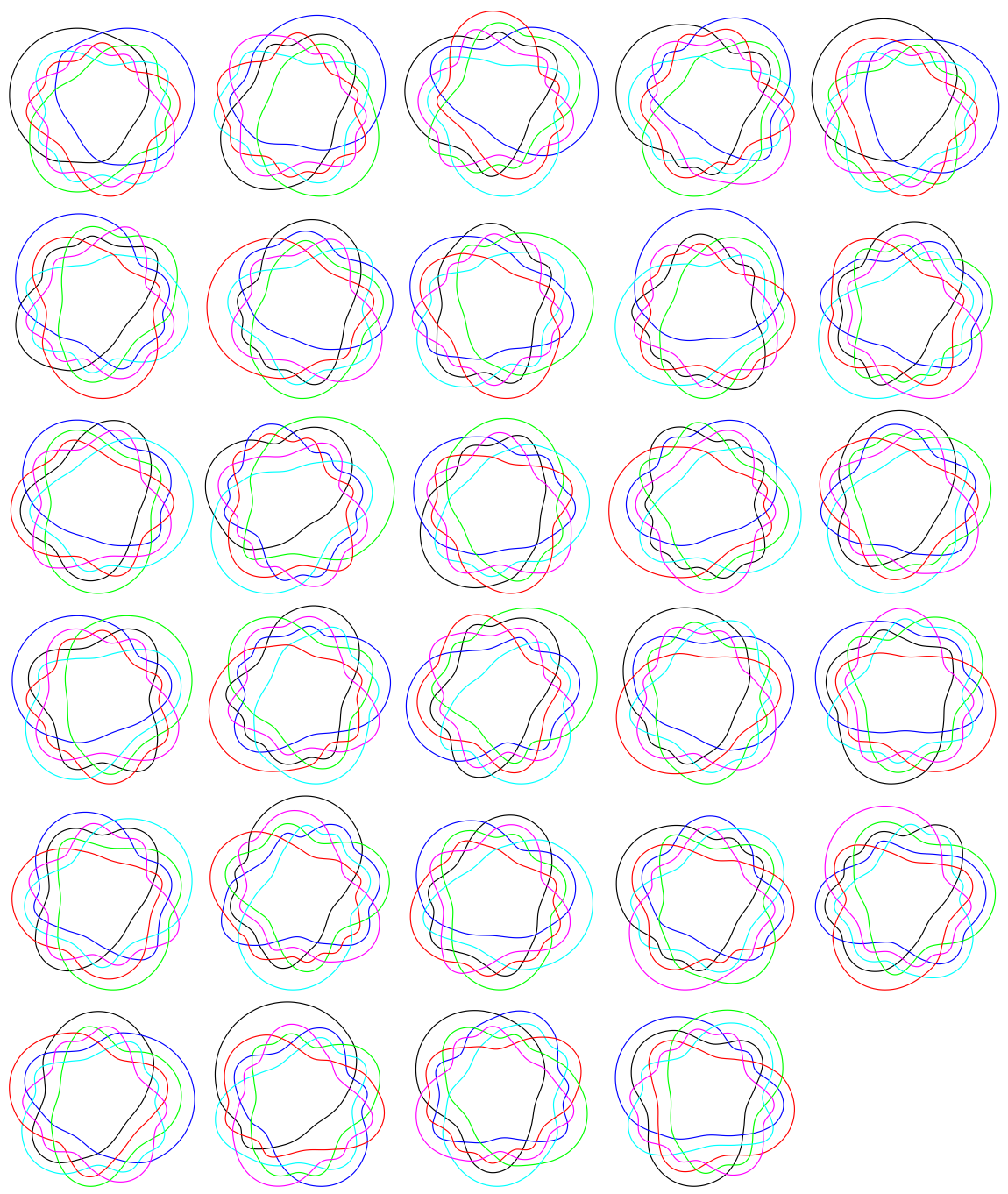

Fig. 9. 29 simple monotone polar symmetric 6-Venn diagrams, representing all possible compositions for the outermost ring. 


\begin{tabular}{|c|c|c|c|}
\hline Composition & Venn Diagrams & Composition & Venn Diagrams \\
\hline 443211 & 25 & 234321 & 10 \\
\hline 4342211 & 0 & 422421 & 0 \\
\hline 3444211 & 38 & 3322421 & 9 \\
\hline 4442311 & 0 & 2442421 & 0 \\
\hline 4333311 & 12 & 3223421 & 3 \\
\hline $\begin{array}{lllllll}3 & 4 & 3 & 3 & 1 & 1\end{array}$ & 9 & 4432231 & 9 \\
\hline 424311 & 0 & 3442231 & 15 \\
\hline 4332411 & 0 & 442532231 & 0 \\
\hline 4422221 & 15 & $\begin{array}{llllll}3 & 3 & 3 & 2 & 3 & 1\end{array}$ & 9 \\
\hline 4333221 & 2 & 322442331 & 0 \\
\hline $\begin{array}{llllll}3 & 4 & 3 & 2 & 2 & 1\end{array}$ & 30 & $4 \begin{array}{llllll}4 & 2 & 2 & 3 & 3 & 1\end{array}$ & 12 \\
\hline 424221 & 0 & $\begin{array}{lllllll}3 & 3 & 2 & 3 & 3 & 1\end{array}$ & 8 \\
\hline 33342221 & 6 & 4222241 & 0 \\
\hline 244221 & 13 & 4322222 & 15 \\
\hline $4 \begin{array}{lllll}4 & 2 & 3 & 2 & 1\end{array}$ & 7 & 42232222 & 22 \\
\hline 34423321 & 8 & 3333222 & 12 \\
\hline 4223321 & 6 & 4222322 & 2 \\
\hline $\begin{array}{llllll}3 & 3 & 3 & 3 & 2 & 1\end{array}$ & 41 & 33323322 & 21 \\
\hline 243321 & 22 & 323232 & 18 \\
\hline 322443221 & 7 & & \\
\hline
\end{tabular}

7. B. Grünbaum, "Venn Diagrams II", Geombinatorics, Volume II, Issue 2, (1992) $25-32$.

8. J. Griggs, C. E. Killian and C. D. Savage, "Venn Diagrams and Symmetric Chain Decompositions in the Boolean Lattice," Electronic Journal of Combinatorics, Volume 11 (no. 1), \#R2, (2004).

9. D. W. Henderson, "Venn diagrams for more than four classes," American Mathematical Monthly, 70 (1963) 424-426.

10. J. Venn, "On the diagrammatic and mechanical representation of propositions and reasonsings," Philosophical Magazine and Journal of Science, Series 5, vol. 10, No. $59,1880$.

11. K. B. Chilakamarri, P. Hamburger and R. E. Pippert, "Venn diagrams and planar graphs," Geometriae Dedicata, 62 (1996) 73-91.

12. F. Ruskey and M. Weston, "A survey of Venn diagrams," The Electronic Journal of Combinatorics, 1997. Dynamic survey, Article DS5 (online). Revised 2001, 2005. 